\title{
HIV Service Interruptions During the COVID-19 Pandemic in China: The Role of COVID-19 Challenges and Institutional Response from Healthcare Professional's Perspective
}

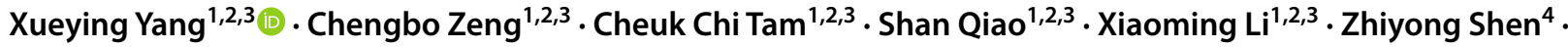 \\ Yuejiao Zhou ${ }^{4}$
}

Accepted: 26 September 2021 / Published online: 6 October 2021

(c) The Author(s), under exclusive licence to Springer Science+Business Media, LLC, part of Springer Nature 2021

\begin{abstract}
During the COVID-19 pandemic, HIV-related services have been unavoidably disrupted and impacted. However, the nature and scope of HIV service disruptions due to COVID-19 has rarely been characterized in China. A cross-sectional online survey was conducted among 1029 HIV healthcare providers in Guangxi, China, from April to May 2020. Latent class analysis (LCA) was first used to identify HIV service disruption levels, then hierarchical multilevel logistic regression was conducted to analyze the relationships of COVID-19 challenges, institutional responses, and HIV service disruption levels adjusting for the clustering effect of institutional ownership levels. Four classes of HIV service disruption were identified, with $22.0 \%$ complete disruption, $15.4 \%$ moderate disruption, $21.9 \%$ minor disruption, and $40.7 \%$ almost no disruption. COVID-19 challenges were positively associated with the probabilities of service disruption levels. Institutional responses were negatively associated with the probabilities of being classified as "minor disruption" and moderated the association of COVID-19 challenges with complete and moderate disruptions compared with no disruption group. To maintain continuity of core HIV services in face of a pandemic, building a resilient health care system with adequate preparedness is necessary.
\end{abstract}

Keywords COVID-19 $\cdot$ HIV service disruptions · COVID-19 challenges · Institutional responses · China

Xueying Yang and Chengbo Zeng have contributed equally.

Xueying Yang

XUEYYANG@mailbox.sc.edu

$\triangle$ Zhiyong Shen

shenzhiyong99999@sina.com

1 South Carolina SmartState Center for Healthcare Quality, Arnold School of Public Health, University of South Carolina, Columbia, SC, USA

2 UofSC Big Data Health Science Center, University of South Carolina, Columbia, SC, USA

3 Department of Health Promotion, Education, and Behavior, Arnold School of Public Health, University of South Carolina, Columbia, SC, USA

4 Guangxi Center for Disease Prevention and Control, Nanning, Guangxi, China

\section{Introduction}

The unprecedented pandemic of coronavirus disease (COVID-19) has triggered not only enormous human casualties and serious economic loss but also significant burdens to the health care system [1]. Despite the fact that the strict containment measures implemented in China had reduced transmission, the healthcare system was largely overwhelmed by such a public health emergency [2]. HIV-related services have been unavoidably disrupted and negatively impacted. The U.S. Centers of Disease Control and Prevention (CDC) posted specific guideline to address concerns and questions of people living with HIV (PLWH) related to their COVID19 risk and prevention [3]. Chinese government issued guidelines related to how to deal with the HIV epidemic in the context of the COVID-19 pandemic [4]. Supported by the Global Fund to Fight HIV, Tuberculosis, and Malaria, a survey of programmes in 106 countries showed that disruptions to service delivery due to the COVID-19 pandemic had affected $85 \%$ of the HIV programmes [5]. 
The COVID-19 pandemic had posed various challenges to maintaining HIV care. First, many public health organizations (especially hospitals) prioritized COVID-19 control over other services including HIV care. Many HIV healthcare providers were reassigned to fight against the COVID19 outbreak because of the shared capacities and logistics with infectious disease responses. As a result, various HIV services were put aside due to the personnel shortage. The ongoing needs of PLWH could not be met [6]. Second, the emergent prevention measures in response to the pandemic, such as stringent quarantine enforcement and transportation lockdown in various cities across China had also severely hindered both HIV patients and healthcare providers from seeking or providing HIV services [6]. Third, HIV clinics may be understaffed due to the lack of personal protective equipment, or absence of safe options/passage for transportation to and from work. Some staffs may be asked to fill in for others who are sick or at high-risk for severe COVID-19. Consequently, these service challenges had led to various service disruptions in HIV clinics [7]. A national anonymous survey conducted by Joint United Nations Programme on HIV/AIDS (UNAIDS) and China CDC reported that 32.6\% of Chinese PLWH were at risk of antiretroviral therapy (ART) discontinuation during the pandemic and $48.6 \%$ did not know where to acquire antiretroviral drugs in the near future $[4,8]$. Moreover, high-risk populations could not get access to pre-exposure and post-exposure prophylaxis (PrEP and PEP) services on time, which might result in a resurgence of new cases of HIV infection [9].

COVID-19 introduced disruptions to HIV service delivery could lead to adverse consequences for PLWH's health beyond those from COVID-19 itself [10-12]. For instance, prediction models from sub-Saharan Africa found that a 6-month disruption of antiretroviral drug supply among 50\% of the population of PLWH who were on ART could lead to a 1.63 times increase in HIV-related deaths over a 1-year period compared to no disruption [13]. In addition, disruption of ART would increase mother-to-child transmission of HIV by approximately 1.6 times [13]. WHO and UNAIDS have also highlighted severe consequences of disruptions, stating that a 6-month disruption of ART could lead to more than 500,000 additional deaths from AIDS-related illnesses [14]. Therefore, empirical data on the depth and breadth of HIV service disruptions (e.g., levels of disruptions and specific services that were disrupted) are warranted. In addition, we need a better understanding of factors that could contribute to the disruption of HIV service delivery, particularly COVID-19 related challenges for HIV service delivery ("COVID-19 challenges").

Institutional responses could possibly moderate the negative effect of COVID-19 challenges on HIV service delivery. In some countries, the COVID-19 pandemic and associated preventive measures (e.g., lockdowns, travel restrictions) have led to increased innovations and ingenuity in HIV service delivery. For example, to ensure the continuity of ART supply, South Africa have implemented several innovative measures, such as extended refills of ART, expanded outof-facility pickup points for refills, transited counselling and adherence support to virtual platforms, and coordinated the local NGOs for ART distribution [15-18]. These institutional responses might play an essential role in building a functional and resilient health systems during COVID-19, but scant literature has examined their effects from a HIV service delivery perspective. What institutional responses have been implemented in Chinese HIV clinics and whether these strategies respond effectively to the HIV service disruptions are unclear. Thus, it is worth investigating institutional responses and their role in moderating the effects of COVID-19 challenges on different levels of HIV service disruptions during the COVID-19 pandemic.

In sum, to improve healthcare delivery and provide sustainable quality care to PLWH during the COVID-19 pandemic and future pandemics, we need a better understanding of the scope of HIV service disruptions and the roles of COVID-19 challenges and institutional responses in exacerbating or alleviating such service disruptions. Specifically, this study aims to explore the levels of HIV service disruptions in China and examine the main and interactive effects of COVID-19 challenges and institutional responses on the levels of HIV service disruptions.

\section{Methods}

\section{Participants and Data Collection}

Data collection of the current cross-sectional study was conducted using an anonymous online survey from a convenience sample of HIV healthcare providers in Guangxi Zhuang Autonomous Region ("Guangxi") from April through May 2020. Guangxi, a region located in Southern China, was consistently ranked third in terms of the number of HIV infection cases across the 31 provinces from 2014 to 2018 [19]. The study design and data collection procedure have been described in detailed elsewhere [20]. The inclusion criteria for participants included: (1) currently a healthcare provider offering HIV-related care and services; (2) 18 years of age or older; and (3) living in Guangxi. First, staff in Guangxi CDC contacted HIV healthcare providers by email. Once the healthcare providers' eligibility was determined, local staff invited them to participate in the online survey. Participants were also encouraged to share the invitation email with their colleagues. An electronic consent was provided prior to the survey started. A total of 1,280 HIV healthcare providers consented and completed the survey. Among them, 251 participants were excluded as they were identified as someone 
from outside of Guangxi ( $n=63$ ), or their responses were considered as random or careless answers $(n=76)$, or outliers on multiple questions $(n=112)$, resulting in a final sample size of 1,029 in this study. Outliers were determined using Mahalanobis distances, which examined if the answers from a participant was away from the centroid for multivariate data. Based on the mean values of institutional responses across disruption levels, post hoc analysis was conducted to test the power in the current sample size [21]. Results revealed that the power is 0.83 and large effect size is 0.27 at $\alpha=0.025$. The research protocol was approved for exempt review by the Institutional Review Board at the University of South Carolina in the United States (Pro00099282) and the ethical approval number from Institutional Review Board of Guangxi CDC is GXIRB2020-33.

\section{Measures}

\section{Institutional Ownership Levels}

Healthcare providers were asked to report the levels of their institutional ownership, which were classified into province-, city-, county-, and community-levels. Due to the small sample sizes (16.6\%) in both province- and city- levels, we dichotomized it into province/city-level and county/ community-level.

\section{HIV Service Disruptions}

Healthcare providers were asked whether there were any disruptions in HIV care services (i.e., HIV clinical service, voluntary counselling and testing [VCT] service, treatment initiation service, outreach work, follow-up service, and ART provision) in their institutes due to COVID-19 using a six-item self-developed checklist (Table 1). The response options for each type of HIV service disruptions were "Yes (1)" and "No (0)". This checklist had good reliability among the study sample (Cronbach's alpha $=0.87$ ).

\section{COVID-19 Challenges}

COVID-19 challenges in participants' institutes were assessed using a self-developed scale comprised of eight items about perceived difficulties and barriers of delivering HIV services during the COVID-19 pandemic. Sample items included "Could not get to work because of self-quarantine or traffic restrictions" and "No manual or guidelines". Response option was rated from "Strongly disagree (1)" to "Strongly agree (5)". A sum score was calculated with higher score indicating a higher level of COVID-19 challenges. This scale showed good reliability in the current study sample (Cronbach's alpha $=0.87$ ).

\section{Institutional Responses}

An 11-item scale was developed in the survey to assess institutional responses to COVID-19 challenges. The scale includes 6-item risk reduction responses for COVID19 (e.g., reduce the occupational exposure to COVID-19 patients) and 5-item institutional responses to HIV services. Given that our outcomes were HIV service disruptions, we used the 5-item subscale of institutional responses in the current study to assess its association with HIV service disruptions. Sample items included "Dispensed antiretroviral medications for 3-6 months at once to ensure ample medications for HIV patients" and "Posted online information about HIV care services". Response option was ranging from "Never (1)" to "Always (5)". A sum score was calculated with a higher score representing a higher level of utilizing these responses during the COVID-19 pandemic. The full scale and two subscales all showed acceptable internal reliability (Cronbach's alpha: 0.75-0.87).

\section{Statistical Analysis}

Data analyses included in this study were latent class analysis (LCA), descriptive statistics, bivariate analysis, and hierarchical multilevel logistic regression. First, LCA was used to identify the disruption levels using the six-item checklist. Based on the standard procedure of mixture modelling [22], an initial, single-group model was specified. Then, successive models with increasing numbers of subgroups were fitted. The final number of subgroups (final model) was determined based on the model interpretation, size of estimated subgroup proportions and model fit indices including Log Likelihood values, Akaike Information Criterion
Table 1 Proportion of different HIV service disruptions $(n=1,029)$

\begin{tabular}{ll}
\hline Items & Yes $(n, \%)$ \\
\hline The HIV clinic service was suspended because of COVID-19 & $52(5.05)$ \\
The VCT service was suspended or postponed because of COVID-19 & $555(53.94)$ \\
The ART application service was suspended or postponed because of COVID-19 & $692(67.25)$ \\
The outreach work was suspended or postponed because of COVID-19 & $351(34.11)$ \\
The follow-up service was suspended or postponed because of COVID-19 & $76(7.39)$ \\
The ART provision was suspended or postponed due to the short supply & $5(0.49)$ \\
\hline
\end{tabular}


(AIC), Bayesian Information Criterion (BIC), adjusted BIC, entropy, $p$-value of bootstrap likelihood ratio test (BLRT). The disruption levels were interpreted based on the itemresponse probabilities by each subgroup and assessed in accordance with prior research. Estimated subgroups with less than $5 \%$ of the total sample were not considered due to the possibility of class over-extraction and poor generalizability [23]. Lower absolute values on the information criterion (i.e., AIC, BIC, adjusted BIC) indicate better fitting models $[22,24]$. The final model was identified when entropy was larger than 0.80 and the $p$-value of BLRT were insignificant.

Second, descriptive statistics were reported on institutional ownership levels, COVID-19 challenges, and institutional responses by the identified disruption levels. Median and interquartile range (IQR) were used to describe continuous variables while frequency and percentage were used for categorical variable. Third, Chi-square test or Kruskal-Wallis test was used to examine the bivariate relationships between variables of interest and disruption levels.

Finally, hierarchical multilevel logistic regression was conducted to predict disruption levels using institutional ownership levels, COVID-19 challenges, and institutional responses. First, institutional ownership level was adjusted in model 1. COVID-19 challenges and institutional responses were entered to model 2 and evaluated their impacts on disruption levels after accounting for institutional ownership levels. Then, an interaction term between COVID-19 challenges and institutional responses was created and entered to model 3. To avoid collinearity, variables were centered before the creation of interaction. The clustering effect of institutional ownership levels was also adjusted during the modelling procedure. Simple slope analysis was used to examine the interactions. LCA was conducted using Mplus version 7.0 (Muthen \& Muthen, Los Angeles, CA) while descriptive statistics, bivariate analysis, and hierarchical multilevel logistic regressions were conducted using "PROC SURVEYLOGISTIC" process in SAS software version 9.4 (SAS Institute, Inc., Cary, NC).

\section{Results}

\section{Latent Class Analysis}

The absolute values of Log Likelihood, AIC, and adjusted BIC decreased with the increasing numbers of subgroups while BIC increased at the 5-class solution as compared to 4-class solution. Additionally, the smallest class at the 5 -class solution included less than $5.0 \%$ of the sample size. Accordingly, the 4-class solution was selected as it accounted for a better classification. Table 2 shows the model fit statistics of each solution in LCA.

Table 3 presents the probability of class memberships. Across the four classes, most HIV service disruption items in Class 4 had the lowest probability compared with other classes and was named as Almost no disruption (420 [40.7\%]). In contrast, items in Class 1 had the highest probability and was named as Complete disruption (226 [22.0\%]). Several items in Class 2 had high probabilities such as HIV clinic service (0.743), VCT service $(0.834)$, outreach service (0.896) and follow-up service (0.754) and was named as Moderate disruption (VCT + treatment initiation service + Outreach service) (158 [15.4\%]). Only two items (outreach service [0.968] and follow up service [0.970]) in Class 3 had high probabilities and was then named as Minor disruption (Outreach service) (225 [21.9\%]).

\section{Descriptive Statistics and Bivariate Analysis}

Among 1,029 healthcare providers, 859 (83.5\%) worked in the county/community-level institutes. The medians and IQRs of COVID-19 challenges and institutional responses were 25.0 (18.0-32.0) and 17.0 (13.0-21.0), respectively.

Chi-square test suggested that institutional ownership levels were significantly associated with disruption levels $\left(\chi^{2}[3]=14.2, p=0.003\right)$. Particularly, $205(90.7 \%)$ of healthcare providers reported complete disruption were from county/community-level institutes. COVID-19 challenges were also significantly related to disruption levels

Table 2 Model fits $(n=1,029)$

\begin{tabular}{llllllll}
\hline Models & LL & AIC & BIC & aBIC & Entropy & BLRT & $\begin{array}{l}\% \text { of the } \\
\text { smallest } \\
\text { class }\end{array}$ \\
\hline 1 & -3976.01 & 7964.02 & 7993.64 & 7974.58 & - & - & - \\
2 & -2910.06 & 5846.11 & 5910.29 & 5869.0 & 0.88 & $<0.001$ & 39.98 \\
3 & -2744.73 & 5529.46 & 5628.18 & 5564.66 & 0.85 & $<0.001$ & 25.22 \\
$4^{*}$ & -2695.01 & 5444.02 & 5577.31 & 5491.55 & 0.86 & $<0.001$ & 16.56 \\
5 & -2670.84 & 5409.67 & 5577.51 & 5469.52 & 0.91 & $<0.001$ & 4.97 \\
6 & -2658.79 & 5399.57 & 5601.96 & 5471.74 & 0.90 & 0.013 & 1.58 \\
\hline
\end{tabular}

$L L$ Log likelihood value

*Model 4 was selected as the final model based on the model interpretation, size of estimated subgroup proportions, and model fit indices 
Table 3 Probability of class membership

\begin{tabular}{|c|c|c|c|c|}
\hline Items & Class 1 & Class 2 & Class 3 & Class 4 \\
\hline Sample sizes $(n, \%)$ & $226(22.0)$ & $158(15.4)$ & $225(21.9)$ & $420(40.7)$ \\
\hline The HIV clinic service was suspended because of COVID-19 & 0.931 & 0.743 & 0.000 & 0.021 \\
\hline The VCT service was suspended or postponed because of COVID-19 & 1.000 & 0.834 & 0.197 & 0.030 \\
\hline The ART application service was suspended or postponed because of COVID-19 & 0.998 & 0.249 & 0.141 & 0.012 \\
\hline The outreach work was suspended or postponed because of COVID-19 & 0.998 & 0.896 & 0.968 & 0.311 \\
\hline The follow-up service was suspended or postponed because of COVID-19 & 0.982 & 0.754 & 0.970 & 0.038 \\
\hline The ART provision was suspended or postponed due to the short supply & 0.961 & 0.291 & 0.279 & 0.040 \\
\hline
\end{tabular}

Class 1: Completed disruption

Class 2: Moderated disruption (VCT + Treatment initiation + Outreach service)

Class 3: Minor disruption (Outreach service)

Class 4: Almost no disruption

$\left(\chi^{2}[3]=279.9, p<0.001\right)$. Participants who reported complete disruption perceived that their institutes had more COVID-19 challenges than those who did not. Bivariate analysis also suggested that the level of institutional responses was related to disruption levels $\left(\chi^{2}[3]=15.2\right.$, $p=0.002$ ). Healthcare providers who reported almost no disruption perceived a higher level of institutional responses in their institute than those who reported somewhat disruptions. Table 4 presents the results of descriptive statistics and bivariate analysis.

\section{Hierarchical Multilevel Logistic Regression}

Using Class 4 as a comparison group, hierarchical multilevel logistic regression was conducted with adjustment of clustering effect of institutional ownership level. Results of model 1 suggested that healthcare providers from county/ community-level institutes had a higher probability to be classified into Class 1 (Complete disruption) (AOR2.332, 95\% CI 2.037-2.671) and Class 2 (Moderate disruption) (AOR 1.477, 95\% CI 1.058-2.062) as compared to those from province/city-level institutes. Model 2 found that COVID-19 challenges were significantly and positively associated with the probabilities of service disruptions (Class 1: AOR 1.226, 95\% CI 1.176-1.278; Class 2: AOR 1.104, 95\% CI 1.079-1.130; Class 3: AOR 1.100, 95\% CI 1.066-1.135). Institutional responses could decrease the probabilities of being classified into Class 3 (Minor disruption [Outreach service]) (AOR0.958, 95\% CI 0.945-0.972) as compared to Class 4 (Almost no disruption).

Finally, in model 3, significant interaction between COVID-19 challenges and institutional responses could be found in the comparison between Class 1 (AOR 0.993, 95\% CI 0.991-0.995), Class 2 (AOR 0.997, 95\% CI 0.996-0.998) versus Class 4. Under the same COVID19 challenges, healthcare providers from the institutes

Table 4 Descriptive statistics and bivariate analysis

\begin{tabular}{|c|c|c|c|c|c|c|c|}
\hline Variables & Total & Class 1 & Class 2 & Class 3 & Class 4 & $\chi^{2}$ & p-value \\
\hline Institutional ownership levels & & & & & & $14.2^{\dagger}$ & 0.003 \\
\hline Province/city & $170(16.5)$ & $21(9.3)$ & $22(13.9)$ & $46(20.4)$ & $81(19.3)$ & & \\
\hline County/community & $859(83.5)$ & $205(90.7)$ & $136(86.1)$ & $179(79.6)$ & $339(80.7)$ & & \\
\hline $\begin{array}{l}\text { Institutional responses (Median, } \\
\text { IQR) }\end{array}$ & $17.0(13.0,21.0)$ & $17.0(13.0,21.0)$ & $17.0(13.0,21.0)$ & $16.0(12.0,21.0)$ & $18.0(15.0,21.0)$ & $15.2^{\ddagger}$ & 0.002 \\
\hline $\begin{array}{l}\text { COVID-19 challenges (Median, } \\
\text { IQR) }\end{array}$ & $25.0(18.0,32.0)$ & $32.0(28.0,38.0)$ & $27.0(22.0,32.0)$ & $26.0(21.0,32.0)$ & $19.0(12.0,26.0)$ & $279.9^{\ddagger}$ & $<0.001$ \\
\hline
\end{tabular}

$I Q R$ Interquartile range

${ }^{\dagger}$ Chi-square test

${ }^{\ddagger}$ Kruskal-Wallis test

Class 1: Complete disruption

Class 2: Moderate disruption (VCT + Treatment initiation + Outreach service)

Class 3: Minor disruption (Outreach service)

Class 4: Almost no disruption 
Table 5 Hierarchical multilevel logistic regressions with adjusting for clustering effect

\begin{tabular}{|c|c|c|c|c|c|c|}
\hline \multirow[t]{2}{*}{ Models and variables } & \multicolumn{2}{|c|}{ Class 1 vs. 4} & \multicolumn{2}{|c|}{ Class 2 vs. 4} & \multicolumn{2}{|c|}{ Class 3 vs. 4} \\
\hline & AOR & $95 \% \mathrm{CI}$ & AOR & $95 \% \mathrm{CI}$ & AOR & $95 \% \mathrm{CI}$ \\
\hline \multicolumn{7}{|c|}{ Model 1: Institutional ownership levels } \\
\hline County/community & $2.332 * * *$ & $2.037-2.671$ & $1.477^{*}$ & $1.058-2.062$ & 0.930 & $0.861-1.004$ \\
\hline Province/city & Reference & & & & & \\
\hline \multicolumn{7}{|c|}{ Model 2: Institutional ownership level } \\
\hline County/rural & $2.232 * * *$ & $1.631-3.053$ & 1.464 & $0.948-2.261$ & 0.928 & $0.839-1.026$ \\
\hline Province/city & Reference & & & & & \\
\hline Institutional responses & 0.965 & $0.929-1.003$ & 0.975 & $0.908-1.047$ & $0.958 * * *$ & $0.945-0.972$ \\
\hline COVID-19 challenges & $1.226^{* * *}$ & $1.176-1.278$ & $1.104 * * *$ & $1.079-1.130$ & $1.100 * * *$ & $1.066-1.135$ \\
\hline \multicolumn{7}{|c|}{ Model 3: Institutional ownership levels } \\
\hline County/community & $2.242 * * *$ & $1.655-3.036$ & 1.472 & $0.947-2.289$ & 0.932 & $0.837-1.037$ \\
\hline Province/city & Reference & & & & & \\
\hline Institutional responses (S) & 0.986 & $0.946-1.029$ & 0.969 & $0.902-1.040$ & $0.949 * * *$ & $0.935-0.964$ \\
\hline COVID-19 challenges (C) & $1.233 * * *$ & $1.194-1.273$ & $1.109 * * *$ & $1.083-1.135$ & $1.103 * * *$ & $1.072-1.135$ \\
\hline $\mathrm{S}^{*} \mathrm{C}$ & $0.993 * * *$ & $0.991-0.995$ & $0.997 * * *$ & $0.996-0.998$ & 0.999 & $0.997-1.001$ \\
\hline
\end{tabular}

Class 1: Complete disruption

Class 2: Moderate disruption (VCT + Treatment initiation + Outreach service)

Class 3: Minor disruption (Outreach service)

Class 4: Almost no disruption

$A O R$ adjusted odds ratio; $C I$ confidence interval

${ }^{*} \mathrm{p}<0.05 ; * * \mathrm{p}<0.01 ; * * * \mathrm{p}<0.001$. $\mathrm{S}{ }^{*} \mathrm{C}$ : The interaction between institutional responses and COVID-19 challenges

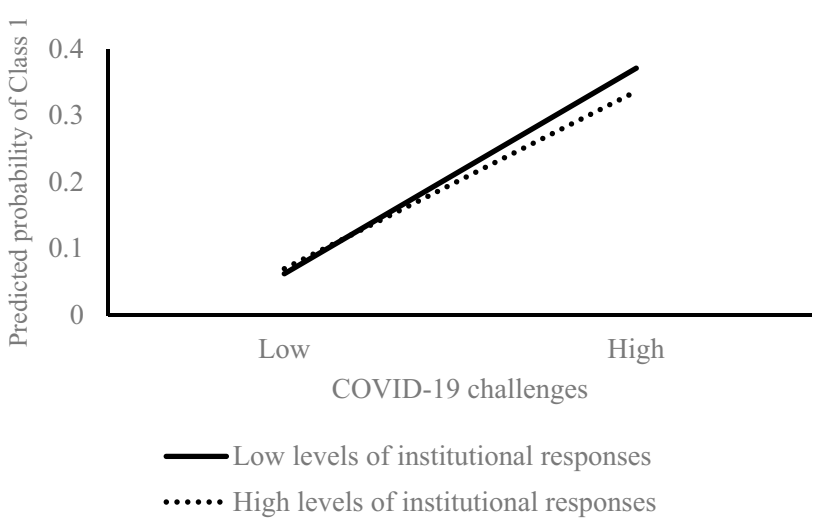

Fig. 1 Interaction between COVID-19 challenges and institutional responses in the comparison between Class 1 (Complete disruption) and Class 4 (Minor disruption). Note Higher and lower are defined as values above or below the mean, respectively

with more institutional responses were less likely to be classified into Class 1 and Class 2. Similar with model 2 , relationships between institutional ownership levels, institutional responses, and disruption levels could also be found in model 3. Table 5 shows the results of hierarchical multilevel logistic regression. Figures 1, 2, and 3 are the results of simple slope analyses.

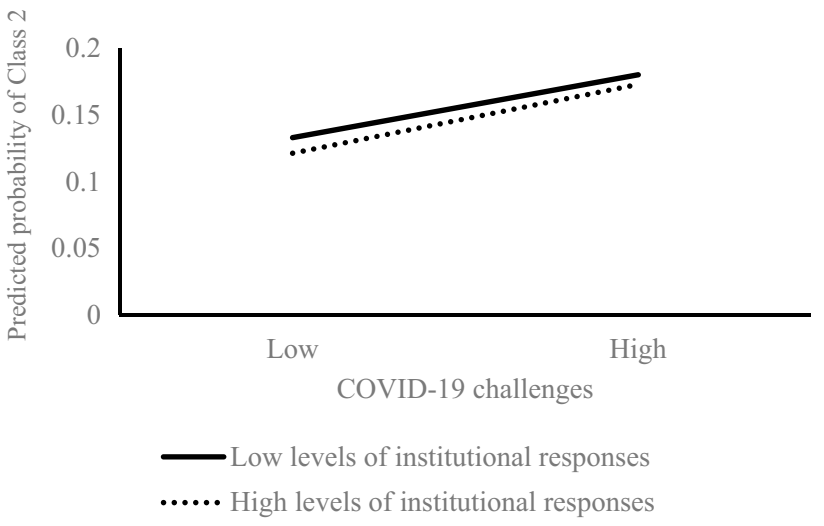

Fig. 2 Interaction between COVID-19 challenges and institutional responses in the comparison between Class 2 (Moderate disruption) and Class 4 (Minor disruption). Note Higher and lower are defined as values above or below the mean, respectively

\section{Discussion}

Using LCA, this study identified four levels of HIV service disruptions in Guangxi China during the COVID19 pandemic, with $22.0 \%$ of healthcare providers classified as working in HIV clinics with complete disruption, $15.4 \%$ classified as working in HIV clinics with moderate 


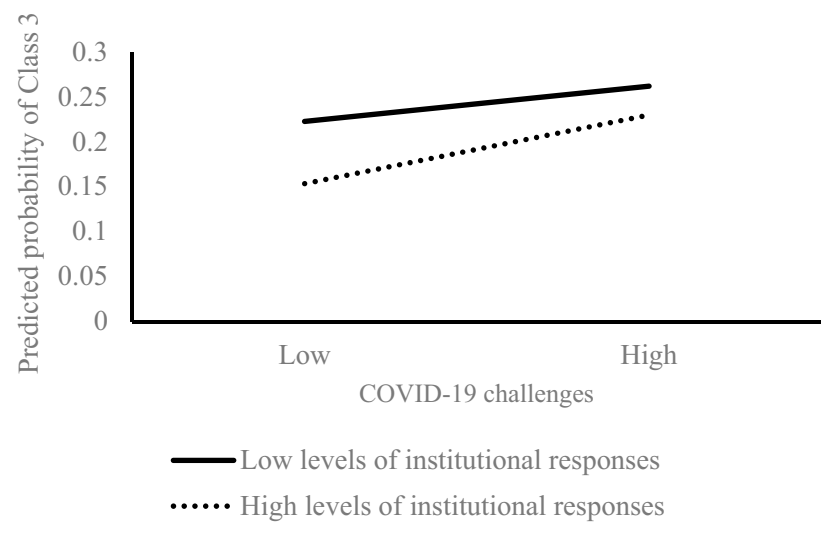

Fig. 3 Interaction between COVID-19 challenges and institutional responses in the comparison between Class 3 (Almost no disruption) and Class 4 (Minor disruption). Note Higher and lower are defined as values above or below the mean, respectively

disruption, $21.9 \%$ and $40.8 \%$ classified as working in HIV clinics with minor or almost no disruption, respectively. Our findings also suggested that healthcare providers who worked in county/community-level institutes were more likely to encounter complete disruption. Such a scope of HIV service disruption is smaller than the findings in a US study [25]. It is possible that the varied pandemic phases and mitigation measures (e.g., magnitude and timing of lockdown) between the two countries account for such a difference. The prevalence of COVID-19 cases was low in Guangxi and the outbreak was quickly controlled due to the quick and stringent mitigation measures from the Chinese government. In the meantime, such a difference could also be explained by the different measure methods. In our study, instead of directly collect information from HIV clinics/facilities, the disruption status was the estimation from healthcare providers. As the sample might not cover all the HIV service facilities, the scope of disruptions might be underestimated. Future studies are warranted to further investigate the geographic disparities of HIV service disruptions in the long term. The HIV service delivery was more heavily disrupted in county/communitylevel institutes than province/city-level even after adjusting for clustering effect, with the difference more evident for institutes with complete disruption. Institutions with more COVID-19 challenges are more likely to have service disruptions in all different degrees. The interaction effects of institutional responses and COVID-19 challenges were significant between the complete vs no disruption and moderate vs no disruption groups after considering clustering effect. As Guangxi was not the hardest hit area of the COVID-19 pandemic, the scope of HIV service disruptions in other areas of China might be more severe than Guangxi. However, the findings from this study might still give us a hint of HIV service disruptions in China because the magnitude of HIV service disruptions is not necessarily overlap with the density of COVID-19 cases [25].

In addition to the summarized disruption levels, our results revealed specific HIV services that were likely to be disrupted during the COVID-19 pandemic. Since HIV viral suppression is critically important, thus many considerations for HIV service delivery in the context of COVID-19 focused on undisrupted ART. Thus, it was not surprising that ART provision or refill services were rarely suspended or postponed in this study. However, around two-third health care providers reported that treatment initiation procedures were suspended or postponed for newly diagnosed HIV infection, which means that lots of newly diagnosed people could not get timely treatment after their diagnosis during the pandemic. Likewise, over half of the healthcare providers reported that the VCT services were suspended or postponed as well, followed by the outreach services. This may be because all these services require face-to-face interactions. To maintain sustainable counselling or outreach services, decentralized delivery of these services are cornerstones of the strategy, as recommended in the US [26]. Decentralized delivery strategy can occur through existing or newly adopted differentiated HIV service delivery models, including community or private pharmacies, home delivery (via HIV-positive peer networks or community health centers), automated lockers, or community pickup points (e.g., post offices).

Results from the multilevel logistic regression demonstrated that the more COVID-19 challenges, the higher odds to have severe HIV service disruptions. Since having a consistent HIV health care was suggested to be associated with HIV-related clinical outcomes (e.g., viral suppression and high CD4 count) [27], overcoming the challenges of HIV service delivery in the context of COVID-19 pandemic would be beneficial for future preparation. To adapt the shortage of personnel, telehealth options, such as phone calls or other virtual options for routine or non-urgent consultations (including HIV adherence counselling), should be considered with careful scrutinize for patient privacy and confidentiality. Similar options can also be considered in place of patient support services typically offered in the community, such as peer support groups and home visits. Implementation of quarantine, social distancing, and community containment measures have reduced access to routine HIV prevention services. Adapting HIV prevention services, such as pre-exposure prophylaxis and HIV testing, may also be considered amidst the pandemic [26]. HIV self-tests may be an option where traditional in-person testing services are temporarily unavailable. Even with availability of HIV self-testing kits in some areas [28], testing remains a big challenge in settings with scarce access to these kits. Thus, additional efforts are needed to augment access and to facilitate testing. For people who are already diagnosed with HIV, 
active patient tracking and tracing services which ensure linkage to care and identify patients late to appointments or medication pick-ups or lost to follow up, should rely primarily on phone calls (requiring up to date contact information for all clients) before resorting to in-person tracking in communities. To support the maintenance of essential HIV service delivery, the healthcare facilities should also have a readily available sophisticated planning to deal with the dual pandemic, develop a manual or guideline for the coordination when task conflict occurs between HIV and COVID-19 service provision.

The findings from our study indicated the effectiveness of institutional responses in moderating the negative effect of COVID-19 challenges on complete HIV service disruption, thus enhancing institutional responses are warranted. The HIV service disruption during the pandemic exposed the fragility of the current health system. To strengthening public health disaster risk management, resilient health system can reduce vulnerability to the public health consequences of emergent crisis [29]. In the aftermath of a health crisis (e.g., COVID-19 pandemic), strong supply chain systems for essential medicines (e.g., ART supply), safe health facilities (PPE), and adequate well-trained health workers would ensure the provision of undisrupted HIV service delivery. During this pandemic, the Chinese National Center for AIDS/STD Control and Prevention issued a notice guaranteeing a free, undisrupted antiretroviral drug supply and released a list of ART clinics for PLWH to refill ART in China [4]. A study conducted in Shenzhen, China has shared successful strategies in fighting concurrent HIV and COVID-19 pandemics [30]. First, they relocated the resources of the hospital and moved the HIV clinics to an isolated clean zone to prevent nosocomial infection. The HIV care and treatment services were conducted in dedicated spaces that were physically separated from areas where COVID-19 patients were being managed. Second, the hospital minimized the number of follow-up visits by extending the medication refills for both HIV and other comorbidities, using express delivery of the medicine, online virtual platforms for counselling and consultations and medical referral, and telemedicine for PrEP and PEP services. In the nonhospital settings, facilities may consider providing services for PLWH and other chronic illnesses in the community to reduce risk of COVID-19 exposure and infection in health facilities, either using community health workers to deliver care or in makeshift clinics in the community [31]. These approaches can be recommended to build the functional resilience of the healthcare system in the future public health crisis to maintain undisrupted HIV service.

Several limitations are worth to note of this study. First, this study was cross-sectional with a lack of longitudinal follow-up. Causal inference could not be established, and the results can only reflect the situation during a short period of the pandemic. Future longitudinal studies are needed to observe the evolving challenges of HIV service disruption since currently the COVID-19 pandemic was under control in China. Second, the information of COVID-19 challenges and service disruptions are collected based on the individual healthcare provider's viewpoint, instead of directly measuring at the institution level. Since the number of participants were not evenly recruited from each institute, the measurement might subject to bias, which may threaten the internal validity of our findings. Third, the scales of COVID-19 challenges and institutional responses in the study were self-developed due to the lack of established measures. These measures may not be replicated as the pandemic evolves and people's experience change. Furthermore, the measure of HIV service disruptions was dichotomized, which might lose some information or underestimate the extent of variation in the magnitude of disruptions. Last, some other institutional responses, such as adequate financing of emergency health service programs, strong health governance and oversight systems, might be useful to mitigate the HIV service disruptions, but not measured in our study. Given a convenience sampling approach, results in the current study may not be generalized to other Chinese regions.

As suggested by our finding, over half of the HIV service institutions in Guangxi China had a certain degree of HIV service disruptions during COVID-19 pandemic, particularly services that requires face-to-face interactions, such as VCT counselling, follow up and outreach services. COVID-19 challenges, such as shortage of personnel and lack of personal protection equipment, largely hinder the HIV service delivery. Institutional responses could marginally moderate the negative effect of COVID-19 challenges on complete HIV service disruptions comparing with no disruptions. To maintain continuity of core HIV services in face of a pandemic, build a resilient health care system with adequate preparedness is necessary, such as adapting to alternative strategies (telehealth, virtual platform, decentralized service delivery) and develop a readily available sophisticated planning (personnel arrangement, guideline or manual for coordination).

Acknowledgements Research reported in this publication was supported by the National Institutes of Health under Award Number R01MH0112376 and National Science Foundation of China (NSFC) [Grant Number 81761128004]. The content is solely the responsibility of the authors and does not necessarily represent the official views of the National Institutes of Health or NSFC.

Funding Research reported in this publication was supported by the National Institutes of Health under Award Number R01MH0112376 and National Science Foundation of China (NSFC) [Grant Number 81761128004]. 
Code Availability Not applicable.

\section{Declarations}

Conflict of interest The authors have no conflicts of interest.

Ethical Approval The research protocol was approved by the Institutional Review Boards at both the University of South Carolina in the United States and the Guangxi CDC in China.

\section{References}

1. Baldwin R, Mauro BWD. Economics in the time of COVID-19. Washington: CEPR Press; 2020.

2. Liu Q, Luo D, Haase JE, Guo Q, Wang XQ, Liu S, et al. The experiences of health-care providers during the COVID-19 crisis in China: a qualitative study. Lancet Glob Health. 2020.

3. CDC. What to Know About HIV and COVID-19. https://www. cdc.gov/coronavirus/2019-ncov/need-extra-precautions/hivhtml (2020).

4. UNAIDS. UNAIDS and China working together during the COVID-19 outbreak to ensure that people living with HIV continue to get treatment. https://www.unaids.org/en/resources/press centre/pressreleaseandstatementarchive/2020/february/20200218_ china_covid19 (2020).

5. Global Fund Survey: Majority of HIV, TB and Malaria Programs Face Distruptions as a Result of COVID-19. https://www.theg1 obalfund.org/en/covid-19/news/2020-06-17-global-fund-surveymajority-of-hiv-tb-and-malaria-programs-face-disruptions-as-aresult-of-covid-19/ (2020).

6. Sun S, Hou J, Chen Y, Lu Y, Brown L, Operario D. Challenges to HIV care and psychological health during the COVID-19 pandemic among people living with HIV in China. AIDS Behav. 2020;24:1.

7. Jiang H, Zhou Y, Tang W. Maintaining HIV care during the COVID-19 pandemic. Lancet HIV. 2020;7(5):e308-9.

8. Guo W, Weng HL, Bai H, Liu J, Wei XN, Zhou K, et al. Quick community survey on the impact of COVID-19 outbreak for the healthcare of people living with HIV. Zhonghua Liu Xing Bing Xue Za Zhi. 2020;41(5):662-6.

9. Power L. COVID-19 has had a major impact on PrEP, sexual behaviour and service provision. https://www.aidsmap.com/news/ jul-2020/covid-19-has-had-major-impact-prep-sexual-behaviourand-service-provision (2020).

10. El-Sadr WM, Justman J. Africa in the path of Covid-19. N Engl J Med. 2020

11. Drain PK, Garrett N. SARS-CoV-2 pandemic expanding in subSaharan Africa: Considerations for COVID-19 in people living with HIV. EClinicalMedicine. 2020.

12. WHO. The potential impact of health service disruptions on the burden of malaria: a modelling analysis for countries in sub-Saharan Africa. 2020.

13. Jewell BL, Mudimu E, Stover J, Ten Brink D, Phillips AN, Smith JA, et al. Potential effects of disruption to HIV programmes in sub-Saharan Africa caused by COVID-19: results from multiple mathematical models. Lancet HIV. 2020;7(9):e629-40.

14. WHO. The Cost of Inaction: COVID-19-Related Service Disruptions Could Cause Hundreds of Thousands of Extra Deaths from HIV. https://www.who.int/news-room/detail/11-05-2020the-cost-of-inaction-covid-19-related-service-disruptions-couldcause-hundreds-of-thousands-of-extra-deaths-from-hivexterna licon (2020). Accessed 27 May 2020.

15. Flowers T. ART services in the time of COVID-19: Adaptations to differentiated service delivery (DSD) models with a focus on those struggling with ART'. Médecins Sans Frontières, South Africa, (IAS webinar). https://www.iasociety.org/Web/WebCo ntent/File/EduFund/COVID-webinars/Session_1_Presentation_ TracyFlowers_SA.pdf (2020). Accessed 21 April 2020.

16. Wilcher R, Akolo C. Five strategies for preserving key populationfocused HIV programmes in the era of COVID-19. https://www. iasociety.org/HIV-Programmes/Cross-cutting-issues/COVID-19and-HIV/Five-strategies-for-preserving-key-population-focusedHIV-programmes-in-the-era-of-COVID-19 (2020).

17. Songo I. Perspectives from Recipients of Care during the COVID19 Pandemic'. CQUIN DSD and COVID-19 Webinar series. http://www.differentiatedcare.org/Portals/0/adam/Content/lZkUq jU6BkO4zqmeniS1tA/File/CQUIN\%20ITPC\%20RoC\%20Per spectives_MASTER\%20FINAL21April-compressed.pdf (2020) Accessed 21 April 2020.

18. Zvandiri A. Adaptation of the CATS model during COVID-19', in 'COVID-19 and HIV: Continuing services with and for marginalised people. https://frontlineaids.org/wp-content/uploads/ 2020/05/COVID-19-and-HIV-Webinar-slides-FINAL1.pdf (2020) Accessed 15 May 2020.

19. Wu Z, Chen J, Scott SR, McGoogan JM. History of the HIV Epidemic in China. Curr HIV/AIDS Rep. 2019;16(6):458-66.

20. Mi T, Yang X, Sun S, Li X, Tam CC, Zhou Y, et al. Mental health problems of HIV healthcare providers during the COVID-19 pandemic: the interactive effects of stressors and coping. AIDS Behav. 2020;1:1-10.

21. Institute for Digital Research \& Education. One-Way Anova Power Analysis/G*Power Data Analysis Examples; 2021. https:// stats.idre.ucla.edu/other/gpower/one-way-anova-power-analysis/.

22. Ram N, Grimm K. Using simple and complex growth models to articulate developmental change: matching theory to method. Int J Behav Dev. 2007;31(4):303-16.

23. Bauer DJ, Curran PJ. Distributional assumptions of growth mixture models: implications for overextraction of latent trajectory classes. Psychol Methods. 2003;8(3):338.

24. Ram N, Grimm KJ. Methods and measures: Growth mixture modeling: a method for identifying differences in longitudinal change among unobserved groups. Int J Behav Dev. 2009;33(6):565-76.

25. Qiao S, Li Z, Weissman S, Li X, Olatosi B, Davis C, et al. Disparity in HIV service interruption in the outbreak of covid-19 in South Carolina. AIDS Behav. 2020;25:1-9.

26. PEPFAR Technical Guidance in Context of COVID-19 Pandemic. PEPFAR's HIV Response in the Context of Coronavirus Disease 2019 (COVID-19). https://www.state.gov/pepfar/coronavirus/ externalicon (2020). Accessed 26 May 2020.

27. Wang Z, Lin HC. Having a consistent HIV health care provider and HIV-related clinical outcomes. Am J Manag Care. 2020;26(7):288-94.

28. Tang W, Wu D. Opportunities and challenges for HIV self-testing in China. Lancet HIV. 2018;5(11):e611-2.

29. Olu O. Resilient health system as conceptual framework for strengthening public health disaster risk management: an African viewpoint. Front Public Health. 2017;5:263.

30. Wang H. HIV care during the coronavirus disease-2019 pandemic in Shenzhen, China. Curr Opin HIV AIDS. 2020;15(6):341.

31. WHO. Maintaining essential health services: operational guidance for the COVID-19 context. June 2020 Geneva. https://www. who.int/publications-detail/10665-332240externalicon (2020). Accessed 4 June 2020

Publisher's Note Springer Nature remains neutral with regard to jurisdictional claims in published maps and institutional affiliations. 